Review

\title{
The histopathological landscape of the major psoriasiform dermatoses
}

\author{
Raluca Balan ${ }^{1}$, Adriana Grigoraș ${ }^{*, 1}$, Diana Popovici ${ }^{2}$, Cornelia Amălinei ${ }^{1}$ \\ ${ }^{1}$ Department of Morphofunctional Sciences I - Histology, "Grigore T. Popa" University of Medicine and \\ Pharmacy, lasi, Romania, ${ }^{2}$ Department - Medicine of Mother and Child, "Grigore T. Popa" University \\ of Medicine and Pharmacy, laşi, Romania
}

\begin{abstract}
Psoriasiform dermatoses represent a wide spectrum of inflammatory conditions, with several major forms represented by psoriasis, as the prototype of this category, followed by pustular psoriasis, Reiter's syndrome, pityriasis rubra pilaris, lichen simplex chronicus and large-plaques parapsoriasis. They create a diagnostic challenge, both clinical and histopathological, because of their complexity and frequent overlapping of the microscopical features. The characteristic histopathological features of psoriasiform reaction comprise extensive hyperkeratosis, with horizontally confluent but vertically intermittent parakeratosis, which alternate with orthokeratosis, thin granular layer, with relative frequent mitoses, uniform elongated and fused rete ridges, edematous superficial papillary dermis, with dilated capillaries, perivascular lymphocytic infiltrate, Munro's microabscesses, and spongiform pustules of Kogoj. Our paper aims to review the histopathology of major form of psoriasiform dermatoses and to emphasize the characteristic microscopical differences between them, for a better approach of the diagnosis as an important key for clinical and therapeutical management. Using the clinicopathological correlations, a thoroughly evaluation of the microscopical features and compartments distribution or special stainings and techniques, the range of differential diagnosis can be decreased and a more accurate diagnostic can be usually achieved. The insights into the pathogenic mechanisms can lead to new therapeutic opportunities targeted to the specific type of inflammatory lesion.
\end{abstract}

Keywords: psoriasis; immune-mediated dermatitis; keratinocyte proliferation; differential diagnosis

\section{Introduction}

Inflammatory dermatoses comprise a large spectrum of clinico-morphological entities, the most common, known as major forms of psoriasiform dermatoses, being: psoriasis, pustular psoriasis, Reiter's syndrome, pityriasis rubra pilaris, lichen simplex chronicus, and large-plaque parapsoriasis (parapsoriasis en grandes plaques). Psoriasis represents the prototype of this category, which can be defined according to inflammation and epidermal changes, the

Received: June 2019; Accepted after review: September 2019; Published: September 2019.

${ }^{*}$ Corresponding author: Adriana Grigoras,

"Grigore T. Popa" University of Medicine and Pharmacy, Iași, 16 Universitatii Street, lasi, Romania.

Email: a grigoras6600@yahoo.com histological mark being regular elongation of rete ridges, due to greater keratinocyte proliferation, partially or totally involving the epidermis [1]. Although the general histological features are shared by most of the inflammatory dermatoses, there are specific microscopical aspects which are pathognomonic for each major form of this disease, which highlight the differences between all inflammatory dermatoses in terms of clinical appearance, pathogenesis and histopathological characteristics related to the quality of scales and the distribution and composition of the inflammatory infiltrate [1].

The aim of our study is to review the main pathogenetic mechanisms and the clinicohistopathological diagnostic features of major form of psoriasiform dermatoses and to emphasize the characteristic microscopical differences between them, for a better 
approach of the diagnosis, as an important key for clinical and therapeutic management.

\section{Pathogenesis}

Psoriasis is considered a multifactorial immune-mediated dermatitis, with a complex and controversial pathogenesis [2], including an autoimmune component, with a variable geographical and ethnical incidence. The pathogenetic mechanisms are under the regulation of the keratinocytes, immune cells, and different cytokines, some of their changes being considered epiphenomena or later events [3].

The most frequent forms (psoriasis, pustular psoriasis, Reiter's syndrome) share common immune-mediated mechanisms, which involve certain human lymphocyte antigens - HLA-B13, HLA-Bw17, HLA-B27, the latter being known as a major factor for the pathogenesis of ankylosing spondylitis, a chronic immune-mediated inflammatory disease with systemic involvement [4] and for Reiter's syndrome genetic component, where the reactive disease is triggered by enteric infections, such as Chlamydia subspecies [1, 3]. The association of psoriasis and arthritis is also described in children, as juvenile psoriatic arthritis (JPsA), a rare disease in which patients can develop besides the above conditions, plantar fasciitis [5]. The genetic mechanisms in psoriasis are supported by the "allelic instability in mitosis" model [1].

Lichen simplex chronicus (LSC) is related to mechanical stimuli which are responsible for the specific pruritus, with secondary lichenification, completed by a psychogenic component [1].

For the parapsoriasis spectrum of diseases, both the relationship between largeplaque parapsoriasis and mycosis fungoides and the possibility for parapsoriasis to be part of cutaneous T-cell lymphoma are related to abnormalities of $\mathrm{T}$-cell proliferation and antigen expression [1].

The considered inflammatory dermatoses are characterized by hyperproliferative epidermis due to keratinocytes hyperactivation and intense inflammatory dermic activity, especially supported by dendritic cells, neutrophils, T lymphocytes, and macrophages $[3,6]$. The abnormal skin activity is managed by various proteins with different cellular activities, which are associated with the pathogenesis of the diseases, such as: signal transducer and activator of transcription (STAT), S100 proteins, Wnt family membrane 5a (Wnt5a) protein, p53/TP53 or tumor protein, enzyme biomarkers, retinoic acid related orphan nuclear receptor gamma (RORy/RORyt), tumor necrosis factor (TNF)like weak inducer of apoptosis (TWEAK), aquaporin-3 (AQP3), galectin-3, and the soluble form of $\beta$-amyloid precursor protein (sAPPa) [7]. The earliest vascular changes observed in psoriasis facilitate the traffic of Th1 lymphocytes subclass in the skin, preserving the psoriatic plaque [3].

Some subpopulations of $\mathrm{T}$ regulatory cells (Tregs), like CD4+CD25high T cell and IL-35, as well as semaphorin $3 \mathrm{~A}$ and neuropilin-1 expressing Tregs, which help to preserve the skin immune tolerance to commensal microbes, are significantly decreased in psoriasis $[3,8]$.

In this regard, it was admitted that the mechanism of the psoriasis can be also related to a dramatic decrease of immunotolerance to cutaneous microorganisms. In this respect, a possible relationship between psoriasis as well as plaque psoriasis with Crohn disease, periodontitis, and Streptococcus pyogenes it was found, conditions related to abnormal immune tolerance to intestinal and oral microenvironment [9].

A recent study demonstrated the impact of microRNA-187 (miR-187, the most dynamic form of the small noncoding RNA molecules) on cellular functions, showing that its downregulation in psoriatic lesions and cytokines-triggered keratinocytes inhibits the cells hyperproliferation through CD276, while themiR-187's overexpression decreases acanthosis, which led to psoriasis outcome improvement in mouse models [10]. In another study, which used quantitative polymerase chain reaction (qPCR), it was emphasized that miR-126 is positively correlated with psoriasis severity index score, its upregulation promoting proliferation and keratinocytes' 
inflammation by suppressing cell apoptosis [11].

Another mechanism which is considered to have a major role in the psoriasis pathogenesis and its related inflammatory skin dermatoses is the IL-1 $\beta-I L-1 R$ signaling pathway which may be involved in the amplification of the skin inflammatory cascade through the regulation of dermal IL-17producing cells and stimulation of keratinocytes [12, 13]. However, plaque and guttate psoriasis show important differences between the phenotype and function of $T$ lymphocytes, IFN- $\gamma$ and IL-17 from CD4+T cells being involved in guttate psoriasis, while IFN-y and IL-17 from CD8+T cells are essential in the pathogenesis of plaque psoriasis $[3,14,15]$.

\section{Clinical forms and patterns}

One of the most important clinical aspects of psoriasis is represented by the evolution as chronic relapsing episodes of remission and exacerbation. The most characteristic form of psoriasis is psoriasis vulgaris, presented as well delimitated individual erythematous lesions, with a thick and silvery scale, sometimes diagnosed by Auspitz sign, in which forced elimination of scales leads to minimal areas of bleeding [1]. Psoriasis vulgaris has a tropism for extensors, due to Koebner (isomorphic) phenomenon (trauma related lesions), while inverse psoriasis involves the flexures, presenting shiny erythema and minor scales [1]. The involvement of the nail leads to thickening and yellow-brown discoloration of the nail plate, known as onychodystrophy. This lesion is rarely located in the mucosa, especially on the glans penis, this localization being related to uncircumcision [1]. Another form is guttate (small) psoriasis, as an acute variant mainly in children, manifested by erupted lesions on proximal extremities and trunk, as an immunemediated reaction between streptococcal $M$ protein and human cutaneous tissues [1]. Erythrodermic psoriasis is manifested as generalized erythema, with important scale shedding and skin vascular dilatation preventing thermoregulation [1].
Pustular psoriasis can be associated with typical plaque-type lesions, as sterile pustules on palms and soles or can be generalized with systemic symptoms, as confluent sterile pustules over the trunk and extremities [1]. Localized psoriasis includes several clinical forms generically known as palmoplantar psoriasis, like: pustular psoriasis of Barber, pustular bacterid of Andrews, acrodermatitis of Hallopeau [1], which share similar histopathological features. The generalized von Zumbusch episodic psoriasis can be associated with hypocalcemia and may be accelerated by systemic corticosteroids, iodides, salicylates, and progesterone [16, 17]. During pregnancy, pustular psoriasis is manifested as impetigo herpetiformis, with a generalized distribution of the lesions [1]. The disease can also evolve with arthritis, erythroderma, or mucous membrane lesions such as fissured and geographical tongue [3]. Several variants of pustular psoriasis were described, as following: acral variant, developed on a background of acrodermatitis continua, exanthematic form, diaper pustular psoriasis, annular psoriasis, linear psoriasis, and localized variant [3].

Reiter's syndrome tend to affect young men, being highlighted by the specific triad of urethritis (with negative urethral cultures), arthritis, and conjunctivitis, which is complete only in one-third of patients. Specific lesions comprise mucocutaneous forms as keratoderma blenorrhagicum (psoriasiform eruption of the palms and soles), balanitis circinata (penile eruption), geographic tongue (ulcerative or erosive oral lesions), and subungual pustules with oncholysis [1]. Moreover, it has been associated with different gut and genital infectious agents, like: Shigella flexneri, Yersinia enterocolitica, Campylobacter jejuni, Chlamydia trachomatis, Ureaplasma urealyticum, and Mycoplasma [3].

Pityriasis Rubra Pilaris (PRP) is a rare chronic papulosquamous disease, with a seborrheic dermatitis-like onset, which evolve toward progressively confluent truncal acuminate follicular papules and perifollicular erythema, associated with areas of uninvolved skin [1]. Other associations involve alopecia, nail modifications, and rarely seborrheic keratosis, different cutaneous malignancies or 
hypothyroidism [3]. While adult forms can undergo spontaneous remission, the children tend to have chronic disease. In addition, human immunodeficiency virus (HIV) positive patients who develop PRP have acne conglobata or hidradenitis suppurativa [18].

Lichen Simplex Chronicus (LSC) (circumscribed neurodermatitis) is related to repetitive mechanical trauma and presents as well-defined papules or plaques, frequently hyperpigmented and always associated with pruritus, involving mostly the lower lateral legs, posterior neck, and flexural areas. A specific pattern is represented by prurigo nodularis, characterized by multiple and symmetrical lesions with dyspigmentation and scarring [1, 3]. It was described an oral variant, benign alveolar ridge keratosis, characterized by white plaques on the maxillary keratinized gingiva, due to traumatic factors [3].

Large-plaques parapsoriasis is defined by Brocq as a group of unrelated idiopathic and asymptomatic diseases of the middle age, with a chronic evolution, which comprises large-plaque (atrophic, retiform, and patchstage mycosis fungoides) and smallplaque parapsoriasis (chronic superficial dermatitis, digitate dermatosis), and pityriasis lichenoides [1], also considered by others as a component of T-cell lymphoma or mycosis fungoides [3, 19]. The disease presents as irregular asymmetrical large erythematous patches and plaques, which involve especially the buttocks, flexures, and breasts, usually being unresponsive to specific treatment [20].

\section{Histopathological features}

Because the skin immune response has limited variants of expression, the histopathological diagnosis of cutaneous inflammatory lesions, including the inflammatory psoriasiform dermatoses, is sometimes difficult to achieve, because of their limited and common landscape [21]. The difficulty is also related to the biopsy type, superficial, with less skin compartments, or profound, which involves more or all skin compartments, for a complete and rigorous microscopical picture. The clinicopathological correlations are also important, as both the histopathological and clinical aspects depend on the age of the lesions [1, 21]. Moreover, it is essential to take in consideration the clinical mimickers of the inflammatory dermatoses and to histologically diagnose the lesion before the systemic therapy because it can affect the specific microscopical landscape of the given condition [22].

In this regard, there are used hematoxylineosin (H\&E) staining, as well as acid periodicSchiff reactive (PAS) staining, for the assessment of the basement membrane and the presence of fungal organisms. Other special stainings are sometimes necessary for the diagnosis of inflammatory dermatoses, such as: fibrin staining (Martius-Scarlet blue), acid mucin staining (colloidal iron Hale's), iron staining (Prussian blue), mast cells staining (toluidine blue), melanin staining (Fontana Masson), elastin staining (Orcein), demonstration of calcium deposits (Von Kossa staining), amyloid staining (Congo red). Bacteria and fungi can also be evidentiated with Gram and Gomori stainings [21]. Immunohistochemistry and electron microscopy have a limited value in diagnosis of inflammatory psoriasiform dermatoses (unless they are important for differential diagnostic with other related conditions), while immunofluorescence is specific for vesiculobullous diseases [21].

The characteristic histopathological features of psoriasis reflect its intermittent character, as follows: extensive hyperkeratosis (Figure 1) with horizontally confluent but vertically intermittent parakeratosis, which alternate with orthokeratosis of basket-weave type, thin granular layer, with relative frequent mitoses, delicate psoriasiform acanthosis, and uniform elongated and fused rete ridges (Figure 2). The superficial papillary dermis is edematous, with dilated capillaries, acting upon the above neutrophilic impregnated granular and spinous layers, which become thinner, together forming the Auspitz sign (Figure 2). About $75 \%$ of the cases present pyknotic neutrophils in the stratum corneum, as Munro's microabscesses, and few form spongiform pustules of Kogoj (aggregated neutrophils in the stratum spinosum). The underlesional superficial dermis presents a perivascular lymphocytic infiltrate (Figure 3), with $\mathrm{T}$ lymphocytes, Langerhans cells, and 
fewer neutrophils, which become more abundant in the advanced lesions, and also characteristic CD11c-positive macrophages situated throughout the basement membrane [3]. Unfortunately, only few lesions are characterized by this classic histological landscape. In early stage or eruptive forms, the changes are nonspecific and located mainly in dermis, with superficial perivascular lymphocytic infiltrate, papillary edema, dilated capillaries, and extravasated erythrocytes (Figure 4), with associated mounded and focal parakeratosis and neutrophilic migration in the overlying almost normal epidermis. The neutrophilic infiltrate is more abundant in eruptive forms than in psoriasis vulgaris (Table 1) $[1,3]$.

There are also described several particular histopathological variants and localizations,

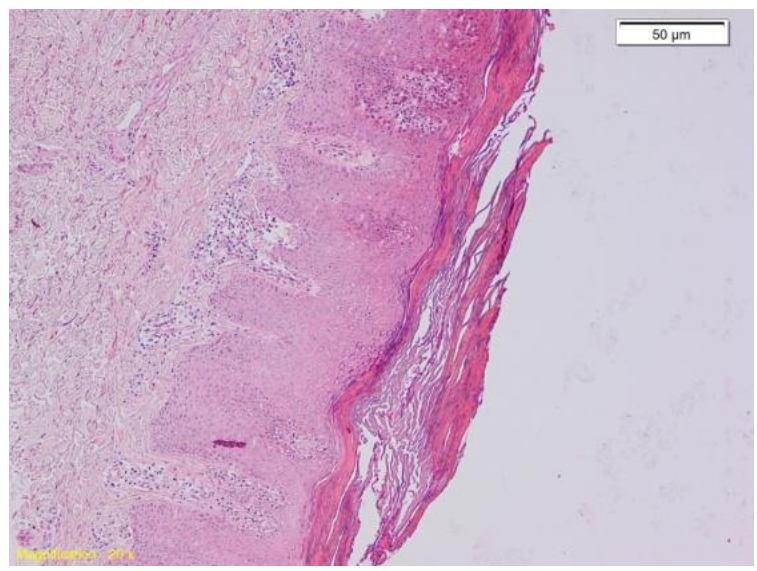

Fig. 1. Psoriasis with extensive hyperkeratosis with horizontally confluent parakeratosis (HE, x50)

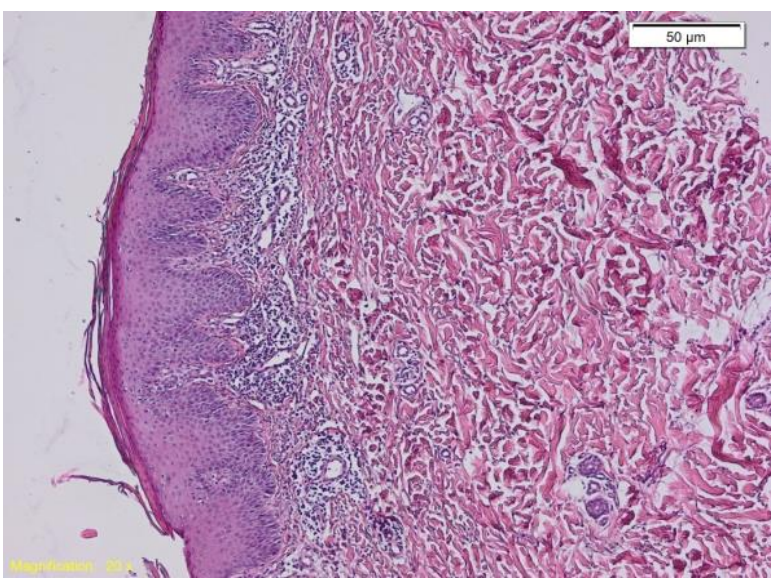

Fig. 3. Perivascular lymphocytic infiltrate in the superficial dermis in psoriasis (HE, x100) some of them overlapping the clinical subtypes, such as: (i) psoriatic neurodermatitis, with pruritic and lichenified plaques situated on the elbows and knees, considered a special form of psoriasis associated with lichen simplex chronicus, (ii) scalp psoriasis, with sebaceous gland atrophy and smaller hair follicles, (iii) nail psoriasis, with hyperkeratosis and spongiosis, (iv) erythrodermic psoriasis, with histological features similar with those seen in incipient psoriasis lesions, ( $v$ ) follicular psoriasis, with parakeratosis follicular plugging and perivascular and perifollicular dermal inflammatory infiltrate, and (vi) annular verrucous psoriasis with exaggerated papillomatosis [3].

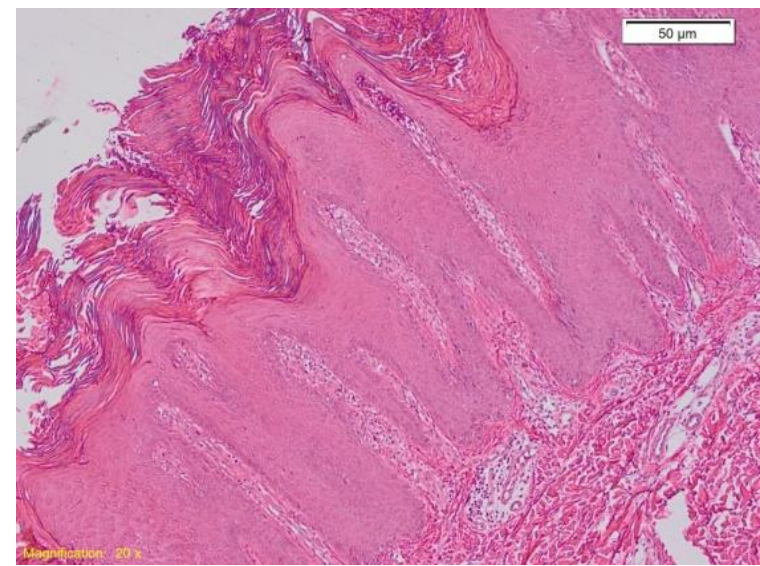

Fig. 2. Psoriasiform reaction consistent with Auspitz sign, with epidermal hyperplasia, parakeratosis, hypogranulosis, elongated and fused rete ridges, edematous papillary dermis, with dilated capillaries (HE, x200)

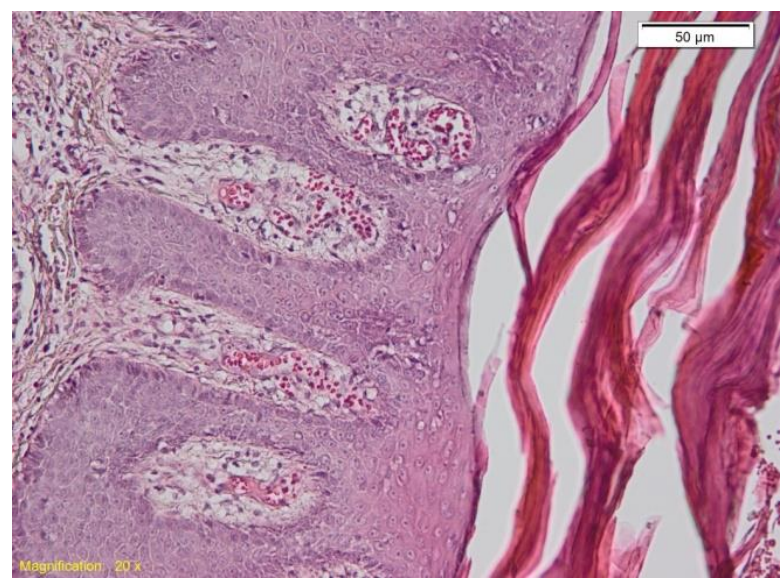

Fig. 4. Early stage psoriasis with superficial perivascular lymphocytic infiltrate, papillary edema, dilated capillaries, and extravasated erythrocytes (HE, $\mathrm{x} 200$ ) 
Pustular psoriasis is microscopically manifested as characteristic spongiform pustule of Kogoj, due to neutrophils migrations from superficial dermal capillaries to intercellular spaces of the edematous Malpighian layer, leading to keratinocytes degeneration and formation of unilocular pustules. The neutrophils finally arrive in the stratum cornosum, forming Munro's microabscesses. The characteristic histological landscape is completed by diffuse parakeratosis, hypogranulosis, regular elongated rete ridges, as well as superficial dermis edema, with capillary dilatation and mixed lympho-neutrophilic perivascular infiltrate, aspects characteristic for psoriasis vulgaris and less developed in eruptive lesions [1]. The palmoplantar pustular psoriasis of Barber and acrodermatitis continua of Hallopeau are histologically manifested as large and unilocular pustules, with spongiform pattern (Table 1) [3].

The histopathological aspects of the Reiter's syndrome clinical triad are almost similar with those met in pustular psoriasis: extensive hyperkeratosis and parakeratosis, thin granular layer, elongated rete ridges, and Munro's microabscesses (Table 1) [1, 3].

Pityriasis rubra pilaris is characterized by horizontal and vertical alternating areas of ortho- and parakeratosis, with particular shoulder parakeratosis (follicular ostia parakeratotic mounds), follicular plugging, irregularly elongated rete ridges, epidermal hyperplasia and hypergranulosis, with infrequent acantholysis, and superficial perivascular lymphocytic infiltrate (Table 1) [1, 3].

In lichen simplex chronicus the histopathologic landscape is highlighted by thickening of epidermis and papillary dermis, characterized by hyperkeratosis, hypergranulosis or acanthosis, irregularly elongated rete ridges, and fibroplasia, with stellate or multinucleated fibroblasts, completed by perivascular lymphocytic inflammation. In the acute form, there is an epidermal spongiosis, without vesicles (Table 1) $[1,3]$.

In parapsoriasis en plaques the histopathologic aspects include epidermal lesions as mild irregular acanthosis, mounding/elevated parakeratosis, focal vacuolar changes, doubled by superficial dermis features manifested as fibrosis, and superficial perivascular lymphocytic infiltrate, characteristic for early stages. In chronic lesions, the picture is completed by band-like dense inflammatory infiltrate, with papillary dermal fibrosis (Table 1) [1, 3].

\section{Differential diagnosis}

There are various conditions which share the main histopathological landscape with psoriasis and related major psoriasiform dermatoses, which are presented below (Table 1) [1, 3, 23].

Psoriasis shares similarities with: (a) other diseases with epidermal neutrophilic microabscesses and pustules (especially in the stratum corneum), like: superficial (dermatophytosis or candidiasis) or deep fungal infections (PAS stain with diastase predigestion), impetigo (includes secondary infections), vasculitis (direct immunofluorescence reveals $\lg G$, $\lg M$ or complement in the superficial vessels), acropustulosis of infancy (subcorneal neutrophilic pustule and perivascular neutrophils and lymphocytes); (b) other papulosquamous diseases with psoriasiform acanthosis, which present as sharp demarcated lesions, with erythema and thick scale, regular acanthosis, clubbed rete ridges, confluent parakeratosis, like: basal cell carcinoma, superficial type secondary syphilis (presents also a deeper plasma cell infiltrate in the dermis), mycosis fungoides, nevus sebaceus, irritated seborrheic keratosis, pityriasis rubra pilaris (usually short epithelial ridges, parakeratosis alternating with orthokeratosis, preserved granular layer, without neutrophilic inflammatory infiltrate), Reiter's syndrome (neutrophils in mounds), chronic eczema (irregular acanthosis), Bowen's disease (atypia), nummular or contact dermatitis (marked spongiosis within rete ridges); (c) lichen simplex chronicus (irregular acanthosis, papillary dermal fibrosis in vertical streaks, fewer neutrophils in stratum corneum). 
Table 1. Clinical and histopathological aspects of the major psoriasiform dermatoses

\begin{tabular}{|c|c|c|}
\hline \multirow[b]{2}{*}{ Type } & \multicolumn{2}{|c|}{ Clinical diagnosis } \\
\hline & Localization & Clinical aspects \\
\hline $\begin{array}{l}\frac{n}{n} \\
\frac{0}{0} \\
\frac{0}{2} \\
0 \\
0\end{array}$ & $\begin{array}{l}\text { Scalp } \\
\text { Groin } \\
\text { Extensors } \\
\text { surfaces } \\
\text { Nails }\end{array}$ & $\begin{array}{l}\text { Sharply circumscribed } \\
\text { plaque with silvery } \\
\text { scale } \\
\text { Auspitz sign } \\
\text { Koebner phenomenon }\end{array}$ \\
\hline
\end{tabular}

\section{Histopathological diagnosis}

Extensive hyperkeratosis with confluent parakeratosis Uniform elongated and fused rete ridges Papillary dermal edema Dilated tortuous capillaries Munro's microabscesses Spongiform pustule of Kogoj

Localized

palmoplantar psoriasis (Barber)

Acrodermatitis continua of Hallopeau Pustular bacterid of Andrews Typical plaque-like lesions von Zumbusch episodic psoriasis Confluent sterile pustules Underlying erythema Oral lesions Precipitated by medications Impetigo herpetiformis (pregnancy)

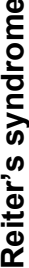

$\begin{array}{ll}\text { Palms and soles } & \text { Triad: } \\ \text { Penile lesions } & \text { Arthritis } \\ & \text { Conjunctivitis }\end{array}$

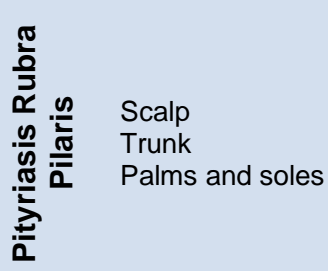

Acuminate follicular papules Erythroderma Palmoplantar keratoderma

Lower lateral legs

Posterior neck Flexures

Prominent pruritus

Well-demarcated

plaques

Lichenification

Excoriation

Hyperpigmentation

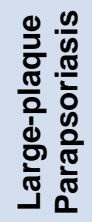

Buttocks

Flexures

Breasts
Scaly erythematous patches and plaques unresponsive to treatment
Hyperkeratosis with confluent parakeratosis Uniform elongated rete ridges

Papillary dermal edema

Dilated tortuous

capillaries

Munro's microabscesses

Spongiform pustule of

Kogoj

Superficial perivascular lymphocytic infiltrate

Vertical and orizontal orthokeratosis and parakeratosis

Shoulder parakeratosis

Follicular plugging

Irregularly rete ridges

Thickened suprapapillary

plates

Superficial perivascular lymphocytic infiltrate

Thicker epidermis and papillary dermis Stellate fibroblasts Superficial perivascular lymphocytic infiltrate Epidermal spongiosis (acute form)

Mild irregular achantosis Mounding parakeratosis Papillary dermal fibrosis Focal vacuolar change Superficial perivascular lymphocytic infiltrate

$\begin{array}{cc}\text { Other dermatoses } & \text { Special } \\ \text { stainings/ } \\ \text { techniques }\end{array}$

Chronic spongiotic dermatitis

Superficial cutaneous

fungal infections

Secondary syphilis

Reiter's syndrome

Lichen simplex

chronicus
PAS stain with

diastase predigestion

Gram and

Gomori

stainings
Dyshidrotic aczema

Pustular drug eruption

Superficial fungal

infection

Subcorneal pustular

dermatosis of
Sneddon-Wilkinson
PAS stain with

diastase predigestion

Gram and

Gomori

stainings
Psoriasis

Chronic dermatitis

Keratosis pilaris

Discoid lupus

erythematosus

Vitamin A deficiency

Seborrheic dermatitis $\quad$ PAS stain with Chronic spongiotic dermatitis

Superficial cutaneous fungal infections

\section{diastase} predigestion

Gram and

Gomori

stainings

\section{Psoriasis}

Guttate psoriasis

Mild chronic dermatitis

Mycosis fungoides 
Pustular psoriasis is more related to dyshidrotic eczema (more spongiotic, with intraepidermal pustules and eosinophilic dermal infiltrate which can indicate also a pustular drug eruption), superficial fungal infections (pustules in the stratum corneum), subcorneal pustular dermatosis of SneddonWilkinson (subcorneal pustules, less parakeratosis and epidermal hyperplasia).

Because Reiter's syndrome and pustular psoriasis share the same histological features, clinical correlations are mandatory. However, in Reiter's syndrome there is more prominent hyperkeratosis and a significant perivascular inflammatory infiltrate.

For Pityriasis Rubra Pilaris, where the clinical aspects are primordial, the differential diagnostic comprises: (a) other papulosquamous diseases (mainly psoriasis), (b) other conditions with follicular plugging (discoid lupus erythematosus with lichenoid inflammation, atopic dermatitis, seborrheic dermatitis, lichen simplex chronicus, ichthyosis vulgaris, keratosis pilaris, lesions of vitamin $A$ deficiency, both with less epidermal hyperplasia), or (c) other diseases with psoriasiform epidermal hyperplasia (Bowen's disease, chronic eczema, clear cell acanthoma, mycosis fungoides which presents atypical epidermotropism, Reiter's syndrome (dilated and tortuous capillaries, neutrophils in mounds).

Psoriasis shares similarities with: (a) other diseases with epidermal neutrophilic microabscesses and pustules (especially in the stratum corneum), like: superficial (dermatophytosis or candidiasis) or deep fungal infections (PAS stain with diastase predigestion), impetigo (includes secondary infections), vasculitis (direct immunofluorescence reveals $\lg G$, $\lg M$ or complement in the superficial vessels), acropustulosis of infancy (subcorneal neutrophilic pustule and perivascular neutrophils and lymphocytes); (b) other papulosquamous diseases with psoriasiform acanthosis, which present as sharp demarcated lesions, with erythema and thick scale, regular acanthosis, clubbed rete ridges, confluent parakeratosis, like: basal cell carcinoma, superficial type secondary syphilis (presents also a deeper plasma cell infiltrate in the dermis), mycosis fungoides, nevus sebaceus, irritated seborrheic keratosis, pityriasis rubra pilaris (usually short epithelial ridges, parakeratosis alternating with orthokeratosis, preserved granular layer, without neutrophilic inflammatory infiltrate), Reiter's syndrome (neutrophils in mounds), chronic eczema (irregular acanthosis), Bowen's disease (atypia), nummular or contact dermatitis (marked spongiosis within rete ridges); (c) lichen simplex chronicus (irregular acanthosis, papillary dermal fibrosis in vertical streaks, fewer neutrophils in stratum corneum).

Pustular psoriasis is more related to dyshidrotic eczema (more spongiotic, with intraepidermal pustules and eosinophilic dermal infiltrate which can indicate also a pustular drug eruption), superficial fungal infections (pustules in the stratum corneum), subcorneal pustular dermatosis of SneddonWilkinson (subcorneal pustules, less parakeratosis and epidermal hyperplasia).

Because Reiter's syndrome and pustular psoriasis share the same histological features, clinical correlations are mandatory. However, in Reiter's syndrome there is more prominent hyperkeratosis and a significant perivascular inflammatory infiltrate.

For Pityriasis Rubra Pilaris, where the clinical aspects are primordial, the differential diagnostic comprises: (a) other papulosquamous diseases (mainly psoriasis), (b) other conditions with follicular plugging (discoid lupus erythematosus with lichenoid inflammation, atopic dermatitis, seborrheic dermatitis, lichen simplex chronicus, ichthyosis vulgaris, keratosis pilaris, lesions of vitamin $\mathrm{A}$ deficiency, both with less epidermal hyperplasia), or (c) other diseases with psoriasiform epidermal hyperplasia (Bowen's disease, chronic eczema, clear cell acanthoma, mycosis fungoides which presents atypical epidermotropism, Reiter's syndrome (dilated and tortuous capillaries, neutrophils in mounds).

Lichen simplex chronicus has histological similarities with other significant pruriginous conditions, other papulosquamous diseases with epidermal hyperplasia, seborrheic dermatitis, chronic fungal infections (more often with neutrophils in the stratum 
corneum), but especially with psoriasis (confluent parakeratosis, neutrophils in mounds, regular clubbed rete ridges, genetic component).

For parapsoriasis, the histopathological features are nonspecific, thus the clinical diagnostic is essential. In early lesions of parapsoriasis, the differential diagnosis comprises superficial perivascular dermatitis or mild chronic dermatitis. Other conditions which share histological similarities with parapsoriasis include: pityriasis rosea (lasts only 6 weeks), pityriasis lichenoides (lasts months to several years, mainly in childhood and young adulthood, with lesions smaller than $5 \mathrm{~mm}$ ), mycosis fungoides (larger plaques, Pautrier microabscesses, pan T-cell markers depletion, atypical T-cell gene rearrangements, and significant epidermotropism).

\section{Conclusions}

The major psoriasiform dermatoses represent complex inflammatory skin

\section{References}

1. Gillum PS, Golitz LE. Psoriasiform Dermatitis. In Barnhill, RL, Crowson, AN (Ed.). Textbook of Dermatopathology. 2nd edition. McGraw-Hill; 2004:61-77.

2. Das RP, Jain AK, Ramesh V. Current concepts in the pathogenesis of psoriasis. Indian $J$ Dermatol 2009; 54(1):7-12.

3. Weedon $D$. The psoriasiform reaction pattern, In Weedon's Skin Pathology. 3rd edition. Churchill Livingstone; 2010:71-91.

4. Chen $\mathrm{B}, \mathrm{Li} \mathrm{J}, \mathrm{He} \mathrm{C}$, et al. Role of HLA-B27 in the pathogenesis of ankylosing spondylitis. Mol Med Rep 2017; 15:1943-1951.

5. Yener GO, Tekin ZE, Yuksel S. Psoriatic fasciitis in a pediatric patient: A case report. World J Clin Cases 2019; 7(1): 69-72.

6. Uttarkar S, Brembilla NC, Boehncke WH. Regulatory cells in the skin: Pathophysiologic role and potential targets for anti-inflammatory therapies. J Allergy Clin Immunol 2019; 143(4):1302-1310.

7. Yadav K, Singh D, Singh MR. Protein biomarker for psoriasis: A systematic review on their role in the pathomechanism, diagnosis, potential targets and treatment of psoriasis. Int J Biol Macromol 2018; 118(Pt B):1796-1810. diseases, which share most of their histopathological features, thus representing a challenge in histopathology.

Using the clinical data, a thoroughly evaluation of the microscopical features and compartments distribution or special stainings and techniques, including molecular markers identification, the range of differential diagnosis can be decreased and a more accurate diagnostic can be usually achieved.

Additionally, clinicopathological correlations may be helpful regarding the disease stage or previous therapies.

Moreover, understanding the pathogenic mechanisms and closely monitoring the development of these major psoriasiform dermatoses can lead to new therapeutic opportunities targeted to the specific type of inflammatory lesion.

\section{Conflict of interest/Funding Statement}

The authors have no conflict of interest to declare.

8. Sabag $A D$, Dias-Polak $D$, Bejar J, Sheffer $H$, Bergman R, Vadasz Z. Altered expression of regulatory molecules in the skin of psoriasis. Immunol Res 2018; 66(6):649-654.

9. Lewis DJ, Chan WH, Hinojosa T, Hsu S, Feldman SR. Mechanisms of microbial pathogenesis and the role of the skin microbiome in psoriasis: A review. Clin Dermatol 2019; 37(2):160-166.

10. Tang L, He S, Zhu Y, et al. Downregulated miR-187 contributes to the keratinocytes hyperproliferation in psoriasis. J Cell Physiol 2019; 234(4):3661-3674.

11. Feng S, Wang L, Liu W, Zhong $Y, X u$ S. MiR126 correlates with increased disease severity and promotes keratinocytes proliferation and inflammation while suppresses cells' apoptosis in psoriasis. J Clin Lab Anal 2018; 32(9):e22588.

12. Cai $Y, X u e ~ F$, Quan, $C$, et al. A Critical Role of the IL-1 $\beta-I L-1 R$ Signaling Pathway in Skin Inflammation and Psoriasis Pathogenesis. $J$ Invest Dermatol 2019; 139(1):146-156.

13. Jaiswal AK, Sadasivam M, Archer NK, et al. Syndecan-1 Regulates Psoriasiform Dermatitis 
by Controlling Homeostasis of IL-17-Producing үठ T Cells. J Immunol 2018; 201(6):1651-1661.

14. Yan $\mathrm{K}$, Han L, Deng $\mathrm{H}$, et al. The distinct role and regulatory mechanism of IL-17 and IFN-Y in the initiation and development of plaque vs guttate psoriasis. J Dermatol Sci 2018; 92(1):106-113.

15. Brembilla NC, Senra L, Boehncke WH. The IL17 Family of Cytokines in Psoriasis: IL-17A and Beyond. Front Immunol 2018; 9:1682.

16. Braverman JM, Cohen I, Black MM. Metabolic and ultrastructural studies in a patient with pustular psoriasis. $\mathrm{Br} J$ Dermatol 1972; 105:189.

17. Shelly WB. Generalized Pustular Psoriasis: Consultations in Dermatology. Philadelphia: Saunders, 1972.

18. Gonzales-Lopez A, Velasco E, Pozo T, Del Villar A. HIV-associated pityriasis rubra pilaris responsive to triple antiretroviral therapy. $\mathrm{Br} \mathrm{J}$ Dermatol 1999; 140:931-934.

19. Simon M, Flaig MJ, Kind $P$, et al. Large plaque parapsoriasis: Clinic and genotyping correlations. J Cutan Pathol 2000; 27:57-60.

20. Lambert WC, Everett MA. The nosology of parapsoriasis. J Am Acad Dermatol 1981; 5:373.

21. Alsaad KO, Ghazarian D. My approach to superficial inflammatory dermatoses. J Clin Pathol 2005; 58:1233-1241.

22. Rohl $R$, Bax $D$, Schierer $S$, Bogner $P N$, Hernandez-llizaliturri F, Paragh G. A case for histologic verification of the diagnosis of atypical psoriasis before systemic therapy. JAAD Case Rep 2018; 4(5):465-467.

23. Rapini RP. Practical Dermatopathology. 2nd Edition. Elsevier Saunders, 2012. 